\title{
Reinhard Schütte
}

\section{Grundsätze ordnungsmäßiger Verbraucherinformationssysteme}

Die Verbraucherpolitik verfolgt das Ziel, ,für die Verbraucherinnen und Verbraucher sichere und selbstbestimmte Handlungsmöglichkeiten zu schaffen." Damit befasst sich die Verbraucherpolitik „mit dem Schutz der wirtschaftlichen Interessen der Verbraucher einschließlich Grundsatzfragen der Verbraucherinformation“"2. Die Verbesserung der Verbraucherposition soll unter anderem durch die „Transparenz, Verständlichkeit und Vergleichbarkeit der Angebote“3 erreicht werden.

\section{Die Informationsproblematik des Verbrauchers}

Die Informationsproblematik des Verbrauchers als Basis dafür, die Entwicklung von Verbraucherinformationssystemen besser zu verstehen, setzt zunächst einen Rahmen voraus, in dem die für Verbraucher relevanten Entitäten und deren Beziehungen enthalten sind: Anbieter, Verbraucher, die Leistung, der staatliche Rahmen (das staatlich-hoheitliche System), Non-Profit-Organisationen, Massenmedien und politisch-rechtliche Institutionen sowie das natürliche System als Umsystem des hoheitlichen Systems (vgl. Abbildung 1). Dabei seien die wesentlichen Sachverhalte skizziert:

- Die Verbraucher und Anbieter stehen sich gegenüber, um eine Leistung von einem Anbieter auf einen

(C) Der/die Autor(en) 2020. Open Access: Dieser Artikel wird unter der Creative Commons Namensnennung 4.0 International Lizenz (http:// creativecommons.org/licenses/by/4.0/deed.de) veröffentlicht.

Open Access wird durch die ZBW - Leibniz-Informationszentrum Wirtschaft gefördert.

1 Bundesministerium der Justiz und für Verbraucherschutz: Aufgaben und Organisation, https://www.bmjv.de/DE/Ministerium/AufgabenOrganisation/AufgabenOrganisation.html (22.12.2019).

2 Bundesministerium der Justiz und für Verbraucherschutz: Abteilung Verbraucherpolitik; Digitale Gesellschaft; Verbraucherrechtsdurchsetzung, https://www.bmjv.de/DE/Ministerium/Abteilungen/Verbraucherpolitik/Verbraucherpolitik_node.html (19.12.2019). Zur Begriffsbasis vgl. P. Kenning: Verbraucherwissenschaften - Begriffliche Grundlagen und Status-Quo, in: P. Kenning, A. P. Oehler, L. A. Reisch, C. Grugel (Hrsg.): Verbraucherwissenschaften. Rahmenbedingungen, Forschungsfelder und Institutionen, Wiesbaden 2017, S. 3-17.

3 Ebenda.

Prof. Dr. Reinhard Schütte ist Professor für Wirtschaftsinformatik und integrierte Informationssysteme an der Universität Duisburg-Essen.
Verbraucher zu einem Preis zu übertragen (marktwirtschaftliche Präsupposition). Dabei sind Verbraucher und Anbieter keinesfalls immer nur in einem direkten Austausch, denn unter anderem aufgrund von Intermediation sind Anbieter nicht nur als einzelne Anbieter zu begreifen, sondern miteinander verwoben. Analoges gilt auch für die Verbraucher, die in eigenen Communities organisiert sein können. Des Weiteren werden die Phasen einer Transaktion unterschieden, die hier als leitgebend für das Informationsbedürfnis von Verbrauchern konzeptualisiert werden. Die Subphasen werden in der Literatur gemeinhin als Anbahnung, Vereinbarung, Durchführung und Anpassung bezeichnet.

- Der Staat definiert mit seinem hoheitlichen Rahmen die Regeln, die sämtliche Akteure mindestens einzuhalten haben, damit es zu einer rechtskonformen Abbildung des Leistungsgeschehens kommt. In diesem Rahmen können allerdings auch Informationen auf freiwilliger Basis zur Verfügung gestellt werden.

- Neben Anbietern und Verbrauchern nehmen Nichtregierungsorganisationen, Stiftung Warentest, Verbraucherzentralen und auch Massenmedien eine bedeutende Rolle im Kontext von Verbraucherinformationen ein.

Bei der aktuellen Gestaltung von Verbraucherinformationssystemen (VIS) ${ }^{4}$ ist zu konstatieren, dass das Informationsmodell des Anbieters dominant ist. In Abbildung 1 wird dies dadurch gekennzeichnet, dass ein Verbraucherinformationsmodell bei der Erstellung eines VIS unter Einhaltung von Gesetzen und Normen traditionell nicht beteiligt ist, was durch die gestrichelte Verbindungslinie ausgedrückt wird. Im engeren Sinn gibt es heute kaum ein VIS, sondern anbieterbezogene Informationssysteme mit Relevanz für den Verbraucher. Das Kernproblem einer solchen Gestaltung von VIS resultiert nicht zuletzt aus der Überzeugung, dass die Vorgaben für die Informationsgestaltung des Anbieters ausreichen können, auch die Anforderungen der Verbraucher an die Gestaltung von Informationssystemen zu berücksichtigen. Die Entwicklung der Leitbilddiskussion in der Verbraucherpolitik hat einen analogen Verlauf genommen: Es gibt nicht den Verbraucher, sondern diese sind zunehmend divers. „Verbraucher und Verbraucherinnen (sind) zumindest in der Wahrneh-

4 Ein Verbraucherinformationssystem (VIS) wird als System verstanden, das aus Informationen als Elementen besteht, die miteinander in Beziehung stehen. 


\section{Abbildung 1}

\section{Gestaltung von Verbraucherinformationssystemen - traditionelle und zweckkonforme Sichtweise}

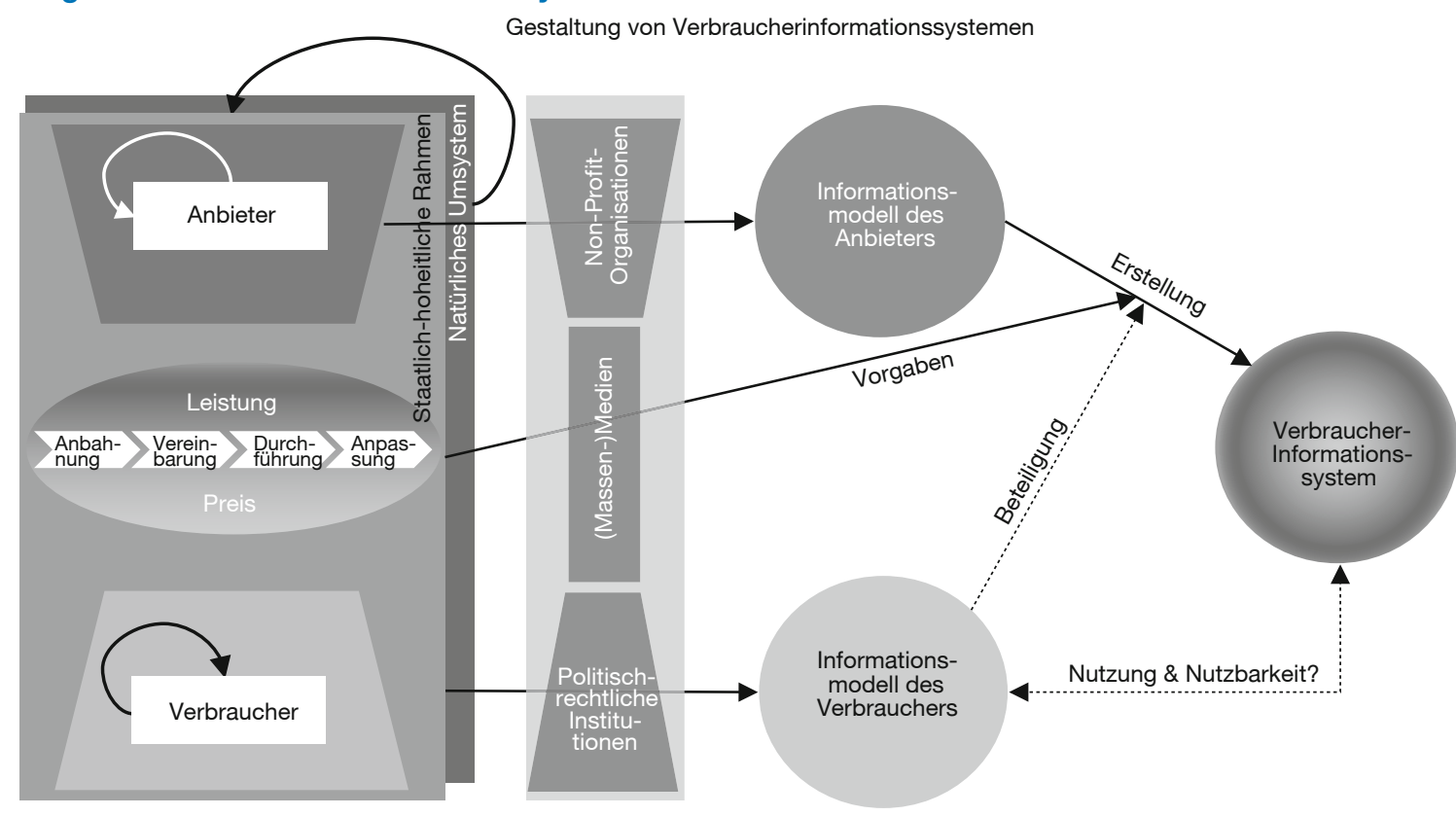

Quelle: eigene Darstellung.

mung der Forschung zusehends agiler, intransparenter, ja unberechenbarer geworden. ${ }^{\text {"5 }}$

Für die Diskussion eines verbraucherorientierten Informationssystems wird Information in Anlehnung an Bateson als „Any difference that makes a difference" ${ }^{6}$ verstanden. Für die Gestaltung eines VIS, das aus der Perspektive eines Verbrauchers konzipiert wird, gilt es, die subjektiven Informationszwecke der Verbraucher zu erfüllen, die nachfolgend skizziert werden:

- Das oberste Gebot ist, dass die Informationen für den Zweck des Verbrauchers - seine jeweilige Handlung in einer der vier Transaktionsphasen - nützlich sind.

- Die Nutzung der Informationen setzt voraus, dass diese sachlich und zeitlich richtig sind.

- Die nützlichen und richtigen Informationen sollten verständlich sein, d.h. sie sollten für den Verbraucher affektiv und kognitiv zu verarbeiten sein.

5 K. U. Hellmann: Leitbilder, Erlebnisse und die mentale Dimension des Konsums. Zum Menschenbild der akademischen Verbraucherforschung, in: C. Bala; W. Schuldzinski (Hrsg.): Jenseits des Otto Normalverbrauchers. Verbraucherpolitik in Zeiten des „unmanagable consumer“, Düsseldorf 2018, S. 19-52; C. Bala, W. Schuldzinski: Einleitung. One Size does not fit it all!, in: dieselben: Jenseits des Otto Normalverbrauchers ..., a.a. O., S. 7-17.

6 G. Bateson: Form, Substance, and Difference, in: General Semantics Bulletin, Nr. 37 (1970), S. 5-13; The Nineteenth Annual Alfred Korzybski Memorial Lecture, New York, 9.1.1970.
- Die Informationen über die Produkte und Leistungen eines Anbieters sollten bezüglich der Nutzung von Leistungen glaubwürdig sein, d.h. die mit der Nutzung verbundenen Erwartungen sollten durch Informationen glaubwürdig kommuniziert werden.

- Aufgrund der vielen unterschiedlichen Sender von Informationen sollte aus Sicht der Verbraucher auch ein Vergleichbarkeitspostulat formuliert werden: Die Ausführungen zu den Leistungen von unterschiedlichen Sendern sind vergleichbar.

- Eine Restriktion über alle zuvor formulierten Gebote bildet die Wirtschaftlichkeit, denn die Bereitstellung und die Nutzung von Informationen durch den Verbraucher sollten wirtschaftlich möglich sein.

Diese Prinzipien bilden nachfolgend die Basis für die Entwicklung der Grundsätze ordnungsmäßiger VIS. Die Überlegung, Grundsätze ordnungsmäßiger VIS zu entwickeln, orientiert sich erstens an den Grundsätzen ordnungsmäßiger Buchführung; ${ }^{7}$ zweitens werden die Grundsätze ordnungsmäßiger Modellierung für die Gestaltung von Informationssystemen herangezogen. ${ }^{8}$

7 Vgl. A. Moxter: Die handelsrechtlichen Grundsätze ordnungsmäßiger Buchführung und das neue Bilanzrecht, in: Zeitschrift für Unternehmens- und Gesellschaftsrecht, 9. Jg. (2009,) H. 2, S. 254-276.

8 Vgl. R. Schütte: Grundsätze ordnungsmäßiger Referenzmodellierung. Konstruktion konfigurations- und anpassungsorientierter Modelle, Wiesbaden 1998 
Grundsätze ordnungsmäßiger

Verbraucherinformationssysteme

Anhand der zuvor skizzierten Zwecke ergeben sich die sechs Grundsätze ordnungsmäßiger Verbraucherinformationssysteme: Jeder Grundsatz wird mit seinen Zwecken formuliert und die verfolgten Ziele sowie Maßnahmen zur Erreichung der Ziele skizziert.

1. Grundsatz der Problemadäquanz: Der erste Zweck, der den Situationsbezug konkretisiert, ist die phasenabhängige Informationsbereitstellung unter Beachtung des Informations- bzw. Schutzinteresses des Verbrauchers (z. B. Nutzen-, Risiko-, Preis- oder Erfahrungsaspekte). Einen zweiten Zweck bildet die Nachhaltigkeit, damit die ökologischen Entscheidungskonsequenzen einerseits und die Verhaltenskonsequenzen der Verbraucher andererseits transparent werden. Das mit dem Grundsatz der Problemadäquanz verfolgte Ziel besteht darin, dass die Nutzbarkeit eines Systems für ein Problem des Verbrauchers erhöht wird. Die Maßnahmen zur Förderung der Ziele und damit zur Erreichung des Zwecks des Grundsatzes der Problemadäquanz sind im Erstellungsprozess die Verbraucherpartizipation bei der Entwicklung des Systems, die Multi-Zweck-Konzeption beim Entwurf des Informationssystems, damit das oder die Systeme für mehrere Zwecke von Verbrauchern verwendet werden können und die Entwicklung und Nutzung validierter Referenzmodelle von VIS, um aus den Erfahrungen von anderen VIS zu lernen.

2. Grundsatz der Richtigkeit: Dessen differenzierte Zwecke orientieren sich an der Richtigkeit der Aussagen über die angebotene Leistung. Somit wäre aus Verbrauchersicht die Forderung nach einer „wahrheitsgemäßen" Aussage zu der Leistung bzw. dem Produkt und auch dem Prozess des Anbieters zwingend. Die sachliche Richtigkeit ist dabei nicht zeitunabhängig zu bewerten, sondern immer in einen Raum-Zeit-Index eingebunden. Diese Forderung an die Information gilt auch für Angaben zur Nachhaltigkeit der Leistungen. Die mit dem Grundsatz der Richtigkeit verbundenen Ziele sind aus den Zwecken abzuleiten und wären korrekte Informationen des Anbieters zu seinen Leistungen gemäß der Vorgaben des Gesetzgebers und des Verbraucherschutzes im allgemeinen, die Transparenz über die Nachhaltigkeit des Anbieterverhaltens für das Produkt und auch des Prozesses, die Transparenz über die Nachhaltigkeit des Verbraucherverhaltens aus Produkt- und Prozesssicht. Die Maßnahmen zur Erreichung der Ziele wären einerseits traditionelle Kennzeichnungspflichten und sonstige Dokumentationspflichten, die dabei auch in dem gesamten Prozess der Transaktion relevant sind.
3. Grundsatz der Verständlichkeit: Der Grundsatz der Verständlichkeit setzt unmittelbar an einer Stelle der Informationsasymmetrie an: der Wissensdifferenz zwischen Anbieter und Verbraucher. Aufgrund abweichender "Sprachspiele“" von Anbietern und Verbrauchern (z.B. der technische Zugang fehlt den Verbrauchern und die Bedienungsanleitung wird von Ingenieuren verfasst) oder dem Umfang der Informationen (Bedienungsanleitungen, Datenschutzbestimmungen, Allgemeine Geschäftsbedingungen) findet keine Informationsaufnahme statt. Die Verständlichkeit ist eine weitere Voraussetzung für Verbraucher, dass es zu einer verbesserten Handlungsfähigkeit der Verbraucher kommt. Bezüglich der Ziele, die aus dem Zweck der Erhöhung eines Verständnisses als Ziele abgeleitet werden können, ist zu fordern, dass die gewählte Sprache, die Kompaktheit der Darstellung und die Art der Repräsentation an den Fähigkeiten der Verbraucher ausgerichtet sind. Die Maßnahmen zur Erreichung der skizzierten Ziele wären die Sprache, die unterschiedlichen Inhaltsumfänge für Verbraucher, die differenzierten Ausprägungen der Inhalte (z. B. Bilder, Videos, Zeichnungen, Texte oder andere Repräsentationen) und die Gestaltung der Benutzeroberfläche.

4. Grundsatz der Glaubwürdigkeit: Bei dem Grundsatz der Glaubwürdigkeit wird der Zweck verfolgt, dass der Verbraucher die Information als glaubwürdig einschätzt. Galt der Sender einer Information in der Vergangenheit aus mangelnder Kenntnis oder aufgrund von Verhaltensproblemen für den Verbraucher als unglaubwürdig, wird seine Information vom Verbraucher kaum verwertet. Die mit dem Grundsatz der Glaubwürdigkeit verbundenen Ziele sind, die Risikobewertung der Information durch den Verbraucher zu verbessern und das Nutzenversprechen (z.B. probiotisch, Wirkung von Haarpräparaten etc.) realistisch einzuschätzen. Die Maßnahmen zur Verbesserung der Glaubwürdigkeit bestehen traditionell im Signalling des Anbieters oder der Bereitstellung von Informationen unabhängiger Institutionen. Beim Signalling sollen freiwillige Angaben (zur Leistung und im Prozess) des Anbieters die Transparenz für den Verbraucher erhöhen. Dies kann auch die Dokumentation umfassen, dass sich der Anbieter um Nachhaltigkeit bemüht hat. Die Rolle von unabhängigen Institutionen zur Zertifizierung, die Stiftung Warentest oder Organisationen verbraucherpolitischen Handelns bieten weitere Möglichkeiten, die Information deutlich glaubwürdiger zu machen.

5. Grundsatz der Vergleichbarkeit: Dieser Grundsatz fordert als übergeordneten Zweck die Vergleichbarkeit von Informationen unterschiedlicher Sender (Medien, Anbieter, unabhängige Organisationen oder auch andere Verbraucher). Aus dem Zweck lassen sich die Ziele für den Grundsatz der Vergleichbarkeit entfalten. Es geht 
zunächst darum, dass die informationsgetriebene Verwirrtheit der Konsumenten reduziert wird und sie damit effizienter Entscheidungen treffen und das Angebot effizienter nutzen können. Die Maßnahmen, die die Zielsituation der Verbraucher unmittelbar verbessern können, sind: Widersprüchlichkeiten in den unterschiedlichen Informationen aufdecken, einheitliche Informationsformate für die unterschiedlichen Sender einführen, allgemeine Bewertungskriterien von Leistungen definieren und einhalten und die Kunstbegriffe und mehrdeutige Inhalte in den Informationen reduzieren.

6. Grundsatz der Wirtschaftlichkeit: Für den Verbraucher wie für andere Wirtschaftssubjekte gilt unter informationsökonomischen Aspekten, dass die Informationen dem Gebot der Wirtschaftlichkeit folgend verfügbar und nutzbar sein müssen. Aus diesem übergeordneten Zweck werden die Ziele abgeleitet, dass sämtliche Grundsätze ordnungsmäßiger VIS dieser Restriktion unterworfen werden. Ausgewählte Maßnahmen zur Verbesserung der Wirtschaftlichkeit von VIS sind: Plattfor- men nutzen, um von den direkten und indirekten Netzeffekten der Verknüpfung vieler Verbraucher und Anbieter zu profitieren, Standards definieren und einhalten, Softwarelösungen integrieren, die im Kontext von VIS relevant sind. Die staatliche Bereitstellung einer Infrastruktur für VIS wäre aus Sicht von Anbietern und Verbrauchern ebenfalls eine Maßnahme zur Förderung der Wirtschaftlichkeit.

Transparenz durch Verbraucherinformationssysteme erhöhen

Die Basis zur Verbesserung der Informationstransparenz für Verbraucher wäre es, wenn die Perspektive von den anbieterbezogenen zu den verbraucherorientierten Verbraucherinformationssystemen wechseln würde. Die aus dieser Perspektive entwickelten Systeme sollten die wesentlichen Zwecke der Verbraucher unterstützen können, wenn sie die skizzierten Grundsätze ordnungsmäßiger Verbraucherinformationssysteme einhalten. Es wäre für die Informationssituation der Verbraucher ein enormer Fortschritt. 